\title{
3 Research Suare \\ Feasibility Trial of an Integrated Treatment "Activate for Life" for Physical and Mental Well-being in Older Adults
}

Melba A Hernandez-Tejada ( $\square$ Melba.A.HernandezTejada@uth.tmc.edu )

University of Texas Health Science Center at Houston https://orcid.org/0000-0003-0732-1248

Alexis Nagel

Medical University of South Carolina

Mohan Madisetti

Medical University of South Carolina

Sundar Balasubramanian

Medical University of South Carolina

Teresa Kelechi

Medical University of South Carolina

Research

Keywords: integrated treatment, Otago Exercise Program, Behavioral Activation

Posted Date: September 7th, 2021

DOl: https://doi.org/10.21203/rs.3.rs-845515/v1

License: (c) (i) This work is licensed under a Creative Commons Attribution 4.0 International License.

Read Full License

Version of Record: A version of this preprint was published at Innovation in Aging on December 1st, 2021.

See the published version at https://doi.org/10.1093/geroni/igab046.3392. 


\section{Abstract}

This paper describes feasibility and patient satisfaction for an integrated treatment to address multiple health outcomes in a sample of older adults living in a low-income independent residence facility and their own homes in the community. Specifically, 30 older adults were offered the opportunity to participate in a feasibility study of different components of Activate for Life treatment targeting balance and physical strength (Otago Exercise Program), breathing retraining (Gentle Yoga and Yogic Breathing), and mental health (Behavioral Activation for Depression). Three treatment combinations were compared in a randomized repeated measures design to determine if adding components to the existing Otago program were feasible and if this affected patient satisfaction. Arm1: the Otago strength and balance program alone ( $n=10)$; Arm 2: Otago + Gentle Yoga and Yogic Breathing $(n=10)$; and Arm 3: Otago + Gentle Yoga and Yogic Breathing + Behavioral Activation (we named this combination 'Activate for Life' $\mathrm{n}$ $=10$ ). . Dependent measures included recruitment rate, session completion characteristics, and satisfaction with the program. Overall, study and treatment components proved feasible, and participants reported high satisfaction with all 3 Arms.

\section{Key Messages Regarding Feasibility}

-What uncertainties existed regarding the feasibility?

Prior to conducting this pilot study, it remained unclear whether combining three different components to address functional, and mental health outcomes in low-income older adults living independently in the community was possible.

-What are the key feasibility findings?

That participants will agree, and will engage in such an integrated treatment. Additionally, findings related to satisfaction with the integrated treatment are key, as are reports that participants continued activities post-treatment.

-What are the implications of the feasibility findings for the design of the main study?

This study demonstrates that combining three different components into one treatment is possible, and that it is possible to implement such an integrated treatment to this, often overlooked and underresourced segment of the population. Second, findings from this study inform recommendations and 'lessons learned' for future applications and improvements of this integrated treatment.

\section{Background}

The current number of older adults ages 65 years and older in the United States (U.S.) is about 54 million (i.e., $16 \%$ of the total) [1]. As the older population grows, challenges related to healthy aging will also intensify, and may diminish the likelihood that one can continue living in one's own homes and 
communities. Unfortunately, many older adults experience physical frailty, declining health, and/or are financially ill equipped to address the physical, psychological, and environmental factors necessary for independent living [2]. In 2019, 4.9 million (8.9\%) older U.S. adults were living below the poverty threshold, with a disproportionately high representation of Hispanic and Black older adults [3, 4]. Healthcare costs or those from an unexpected illness, and physical and mental health deterioration can result in a downward spiral in independent functioning, eventually leading to unwanted institutionalization [5]. "Aging in Place" is an increasingly popular concept that reflects the overwhelming preference of older adults to live out their lives in their own homes as opposed to a structured, typically healthcare focused (and almost universally more expensive) institutionalized setting [6]. This along with the potential social and economic burden of expanding institutionalized care for millions of older individuals is driving research and community efforts that will enable older people to remain in their homes rather than transitioning to care facilities. Successful initiatives are likely to include strategies that address environmental and situational factors that allow older adults to maintain their autonomy while sustaining, or even improving, the quality of their social connections [7].

Age-related physical challenges such as chronic pain, fatigue, and concomitant depression (which is often amplified by pain and fatigue) are quite common in older individuals and are considered significant barriers to 'aging in place'. About $28 \%$ of older adults suffer from chronic pain [8] while 31\% report experiencing significant fatigue [9].Chronic pain and fatigue can result in reduced physical activity, leading to decreased muscle tone and increased risk of injury from accidental falls; intensified arthritisrelated impairment; and other conditions that reduce capacity to participate in activities of daily living or in recreational activities that sustain mental and physical well-being $[10,11,12,13]$. Interventions to address pain and fatigue can be effective with older adults, particularly those targeting mobility and psychosocial $[14,15]$. However, these programs are not always available or accessible to older adults. This is especially true for low-income older adults due to logistic, financial, and geographic barriers [16, 17]. Moreover, many older adults may require more than simple strengthening programs to maintain healthy aging, for instance specific strategies or integrated treatments to improve mental health and engagement in addition to physical health. Very few health-mental health integrated 'aging in place' programs exist in the U.S. and even fewer have been evaluated for their impact on physical and psychological outcomes.

Home-based telemedicine technology may increase access to care and the ability of older adults to 'age in place'. Unfortunately there is a paucity of literature regarding the use of mHealth (e.g., tablet based applications) and eHealth (e.g., televideo via tablet) as a platform for managing pain in older adults [18, 19]. However, technology-based interventions to improve health outcomes in older adults are emerging and seem to improve engagement with providers while addressing barriers to care, with the same effectiveness as traditional settings (in office visits) [20, 21, 22]. To advance this important area of research, this pilot study evaluated the feasibility (as determined by measures related to recruitment, enrollment, retention, and satisfaction with treatment) of combining mindfulness (Gentle Yoga and Yogic Breathing, or "GYYB") [23] and mental health components (Behavioral Activation for Depression, or "BA") [24] with an existing, evidence-based muscle strengthening and balance retraining program, Otago ("OG"), 
endorsed by the Centers for Disease Control as an effective fall intervention program [25]. The overarching goal of the multicomponent $\mathrm{OG}+\mathrm{GYYB}+\mathrm{BA}$ intervention, Activate for Life, is to reduce pain and fatigue in lower-income older adults, ultimately improving overall physical functioning and mental health and increasing the likelihood that older persons are able to successfully 'age in place'.

\section{Methods}

Participants. Older adults ages 60 years and older living in subsidized housing facilities or their own homes in the US Southeast were recruited via word of mouth, flyers placed in housing facilities, or through direct referral by service providers familiar with the pilot program. Interested participants initiated contact or requested that more information be presented by the study team at their facility. Enrollment was offered for those meeting the following inclusion criteria: English-speaking, any gender, aged 60 years or older, residing in low-income housing or meeting the definition of low-income (defined as $\leq 150 \%$ of the official poverty threshold), and experiencing a pain score of $\geq 8$ on the PROMIS Pain Interference short form. Excluded from participation in the study were those who had significant cognitive impairment or dementia (a score between $0-2$ as measured by the Mini-Cog [26]; those unable or unwilling to give consent; those with a physical disability resulting in an inability to ambulate 150 feet (ft.) with or without the assistance of another individual or assistive device; and those who were unable/did not want to operate the provided tablet device. All screening questionnaires were administered following informed consent.

Randomization. For this 12-week pilot trial, we employed a randomized trial design and $3 \times 3$ repeated measures (treatment $x$ time) approach to compare feasibility measures among participant groups in Arm $1(\mathrm{OG})$, Arm 2 (OG + GYYB), and Arm 3 (OG + GYYB + BA; together, these comprise the full "AFL" intervention). After signing the informed consent document, 30 participants were randomly allocated to one of the 3 arms ( $n=10$ each) using a randomization scheme developed by the study biostatistician under the Research Electronic Data Capture (REDCap) system. Study data were obtained at baseline, post-treatment (12-weeks), and at 3-month follow-up.

Procedures. We focused feasibility on four main areas: recruitment, enrollment, retention, and patient satisfaction with treatment. For recruitment, we measured the number of completed treatment sessions completed by participants after treatment initiation. The expected number of sessions to be completed was 12 over the 12 weeks of treatment. We also measured the number of participants who successfully completed the 12-week treatment. The expected number of completers was about $70 \%$ of the participants. This percentage is based on evidence of normal dropout from clinical research in the integrated components $[27,28,29]$. For dropouts, the average session number at which participants decided to withdraw from treatment was noted. A 27-item Patient Satisfaction with Treatment survey was a developed specifically for this study, with yes/no questions, and 5-point Likert scale ranging from not at all satisfied to very satisfied, with subsequent open questions to clarify their yes/no responses. Questions specifically targeted participants' experiences with each component of the assigned Arm, the tablets, software, blood pressure monitoring and activity tracking. We used this same instrument for surveying 
participants assigned to each Arm. The instrument was pre-tested prior to use in the study. This study design was approved on June 5, 2018 by the Medical University of South Carolina Institutional Review Board, approval \#Pro00076835 and registered at ClinicalTrials.gov Identifier NCT03853148.

Data Collection and Analysis. Study data were collected and managed using Research Electronic Data Capture (REDCap) tools. Descriptive methods were used to characterize the data, including percentage distributions, means and standard deviations. Open-ended responses related to their experience with treatment were categorized by the contents of treatment and devices used.

\section{Results}

Demographics. Fifty-nine older adults ages 60 and older were approached and screened for this pilot study. Thirty met the inclusion criteria and agreed to be randomized in equal proportions to the three conditions and nineteen participants completed treatment. Table 1 presents demographic information for the 30 participants who were randomized to the three study arms. The average age of the full sample was 70.6 years $(S D=6.4$ years). The majority of the participants were female, Black, and retired with some college-level education. Most reported taking anti-hypertensive, cholesterol, and/or pain medications. None of the participating older adults had experience with any of the three treatment components. 
Table 1

Participant Demographics and Other Characteristics

\begin{tabular}{|llllllc|}
\hline & Arm 1 & \multicolumn{3}{c}{ Arm 2 } & \multicolumn{3}{c|}{ Arm 3 } \\
\hline Variable & M & SD & M & SD & M & SD \\
\hline Age (years) & 73.4 & 5.6 & 68.5 & 7.5 & 69.8 & 5.4 \\
\hline Variable & $\mathrm{n}$ & $\%$ & $\mathrm{n}$ & $\%$ & $\mathrm{n}$ & $\%$ \\
\hline Sex & & & & & & \\
\hline Female & 7 & $70 \%$ & 7 & $70 \%$ & 7 & $70 \%$ \\
\hline Race & & & & & & \\
\hline White & 5 & $50 \%$ & 5 & $50 \%$ & 2 & $20 \%$ \\
\hline African American & 5 & $50 \%$ & 5 & $50 \%$ & 8 & $80 \%$ \\
\hline Education & & & & & & \\
\hline Some College or More & 6 & $60 \%$ & 6 & $60 \%$ & 5 & $50 \%$ \\
\hline Employment* & & & & & & \\
\hline Retired & 8 & $80 \%$ & 6 & $60 \%$ & 5 & $50 \%$ \\
\hline Medication use & & & & & & $50 \%$ \\
\hline Antihypertensive & 5 & $50 \%$ & 8 & $80 \%$ & 5 & $50 \%$ \\
\hline Cholesterol & 6 & $60 \%$ & 5 & $50 \%$ & 8 & $80 \%$ \\
\hline Pain & 7 & $70 \%$ & 4 & $40 \%$ & 3 & $30 \%$ \\
\hline *Employment category & & & & & & \\
\hline
\end{tabular}

Feasibility. To recruit and enroll participants in this study, we used IRB approved flyers and presentations, as well as word of mouth (e.g., presentations at local senior centers). These strategies were implemented by study personnel at sites where low-income older adults frequent or live. Figure 1 shows the Consort diagram for this study. We approached 59 potentially-eligible older adults, of which 10 were not interested and were interested but were unable to be recontacted for prescreening. We prescreened the remaining 43, 5 of whom were ineligible. Of the remaining eligible candidates 3 decided they were not interested and 5 were unable to be recontacted. The consenting and enrollment processes were initially conducted in person by study personnel at patient residence or recreational sites, and then moved to an online platform (first contact via telephone, then televideo calls were established to complete consent and baseline processes) due to pandemic restrictions. Both approaches were found to be feasible and acceptable by study participants. 
The highest rate of retention was observed for Arm 1, with $70 \%$ of the participants completing treatment. While overall retention was lowest for Arm 3, it is important to note that $80 \%$ of the participants were retained past session 7 , with $50 \%$ completing the full treatment (session 12), despite the fact that involvement in the full AFL intervention required far more effort on the part of the participant. Most Arm 3 participants were able to complete about half the treatment sessions before dropping out, in contrast to dropouts from Arms 1 and 2 who exited the study shortly after completing baseline due to multiple schedule conflicts. Additionally, participants in Arm 3 encountered several unique circumstances that participants in the other two arms did not experience. Unfortunately, one patient died during treatment (after completing visit 7, due to a pre-existing condition) and another participant dropped out after visit 7 due to ongoing issues with technology (poor network connectivity in their location,). Finally, three participants dropped out due to schedule conflicts - two at visit 6 and one at visit 11. Table 2 provides information about the number of completed treatment sessions for each arm.

Table 2

Quantitative Feasibility Outcomes Regarding Treatment Completion

\begin{tabular}{|llllll|}
\hline $\begin{array}{l}\text { Treatment* } \\
\text { Condition }\end{array}$ & $\begin{array}{l}\% \\
<2 \\
\text { visits }\end{array}$ & $\begin{array}{l}\text { 3-6 } \\
\text { visits }\end{array}$ & $\begin{array}{l}7-12 \\
\text { visits }\end{array}$ & $\begin{array}{l}\text { \% with follow- } \\
\text { up }\end{array}$ & Reason for dropout \\
\hline Arm 1 & $20 \%$ & $0 \%$ & $80 \%$ & $70 \%$ & Conflict of schedule \\
\hline Arm 2 & $40 \%$ & $0 \%$ & $60 \%$ & $60 \%$ & $\begin{array}{l}\text { Conflict of schedule, } \\
\text { pain }\end{array}$ \\
\hline Arm 3 & $0 \%$ & $20 \%$ & $80 \%$ & $40 \%$ & $\begin{array}{l}\text { Death, pain, } \\
\text { device(s) issues }\end{array}$ \\
\hline *Arm 1: Otago, Arm 2: Otago + GYYB, Arm 3: Activate for Life & \\
\hline
\end{tabular}

Satisfaction. Satisfaction questions included specific queries about the characteristics and components of the intervention and associated technologies, such as using electronic devices (i.e., activity tracker, blood pressure device, tablet). Overall, $85 \%$ of Arm 1 participants reported high satisfaction with the physical exercise program as compared to $67 \%$ in Arms 2 and 3 . Specifically, participants in Arm 3 reported positive experience with the treatment and interaction with the research team, nonetheless, some participants commented that it did require effort to organize their time so that they could incorporate all of the activities. Another relevant comment was regarding the age difference between the therapist and the clients, insofar as some clients thought that the 50-year age difference between the therapist and patients was too large. Participants who dropped out from treatment reported they were unable to continue for personal reasons rather because of any characteristics of the integrated treatment. Finally, participants in Arms 2 and 3 reported lower satisfaction with the GYYB component. From our interview findings, participants opted not to answer the question regarding GYYB experience. There was an overall lack of engagement with GYYB and patients had difficulty remembering the GYYB exercises when they were asked about their experience with them, likely because they were not performing the activity daily. 
The majority of the older adults in this study reported a low level of experience operating a tablet device. For example, $85 \%$ of Arm 1 participants and $67 \%$ of participants in both Arm 2 and Arm 3 indicated they did not have experience with an iPad/tablet. Learning how to use the iPad/tablet (ease of use) was largely reported to be easy, with $57 \%$ of Arm 1 participants rating the tablet as "easy" or "somewhat easy" to use and $42 \%$ "somewhat difficult" (in Arm 2, 50\% reported that the tablet was "easy" to use versus "somewhat difficult," while in Arm $383 \%$ of participants rated the tablet as easy). The majority of the participants in this study (57\% of Arm 1, 83\% of Arm 2, and 33\% of Arm 3) encountered at least one issue while using the iPad/tablet. Most issues were related to connectivity or resetting the tablet device so that the activity tracker could upload/sync data correctly. Some reported that once they were able to sort out the issues they were "ok" with the device, whereas others felt that it was too much trouble and was part of the reason for dropout. Finally, most participants found the blood pressure monitor "easy" to use (100\% in Arm 1 and $83 \%$ in both Arms 2 and 3) while responses regarding issues encountered using either the blood pressure monitor or the activity tracker (29\% in Arm 1, 67\% in Arm 2, and 50\% in Arm 3) varied across arms. Most issues were related to the device battery life; not understanding the screen of the blood pressure device; skin irritability from the activity tracker, requiring participants to switch from one arm to the other; discomfort; and problems syncing with app (the most commonly reported issue).

Continuing treatment. The majority of the participants indicated they continued performing the OG physical activities they learned during the intervention after completion of treatment ( $71 \%$ in Arm 1,67\% in Arm 2, and 75\% in Arm 3). Participants in all three arms reported exercising an average of twice a week. None of the participants in Arms 2 and 3 continued performing GYYB exercises.

Finally, the research team asked open-ended questions of all participants to describe what they thought were the best and worst parts of the program. Positive aspects included learning about their own ability to challenge themselves, learning how to use the iPad, and ability to self-monitor their own blood pressure. They also expressed a generally positive attitude about being required to log in to the tablet every day to complete tracking activities and blood pressure measurement. They reported that they were motivated and encouraged to complete the intervention because other participants or residents from the same community were involved in the study and this was another way to build friendships. Some reported that initially they did not want to exercise but that once they started participating they wanted to continue. In Arms 2 and 3 there appeared to be a lack of engagement with the GYYB exercises by some of the participants. Verbal reports of participants indicated difficulty understanding the purpose of the GYYB exercises and feeling that they would have liked to see videos that were more targeted to them (for instance, another older adult demonstrating the exercises). Participants also indicated they did not like the writing activities that were part of BA. Specifically, some participants disliked writing down daily activities because they did not want other people (including the therapist or anyone on the study team) to know what they were doing. Also, some had stopped writing due to complications from arthritis and being asked to do this activity was physically challenging. Some participants indicated that it was challenging to complete exercises, particularly when pain was present, or that it was difficult to find motivation to do the exercises on their own. Wearing the activity tracker was uncomfortable for some, and difficulties syncing with the app due to connectivity issues introduced additional challenges. Finally, 
we collected saliva to determine changes of cortisol and other inflammatory levels and patients did not have report any difficulties with data collection procedures. However, at least four reported having difficulty producing the amount of saliva required due to dry mouth, but despite this, all participants had no problems with being requested to do this part of the study.

\section{Discussion}

Older adults living in low-income communities face tremendous economic and health-related challenges that diminish their ability to 'age in place' [30]. Insufficient access to care, particularly in rural areas, exacerbates these challenges and their effects on health. Advances in technology may help to overcome these barriers through increased access to integrated mHealth and physical health interventions focused on improving mood, mobility, and overall well-being [31] that negatively affect 'aging in place'. The present pilot study examined the feasibility of introducing varied levels of integration for existing interventions addressing physical [32], functional [33], and psychological [34] factors that might help independently residing, albeit resource-poor older adults, successfully age in place. We hypothesized that this integrated treatment would not only satisfy our feasibility criteria related to recruitment, enrollment, and retention, but would also be viewed as particularly welcome when delivered via telemedicine, with patients reporting high satisfaction with the program. Our results support the feasibility of the mHealthdelivered intervention and its component parts, insofar as lower income older adults agreed to be randomized to condition, accept conditions of treatment components, and complete 6 or more sessions. Satisfaction with the mHealth component of the Activate for Life intervention was also high.

Overall, about three quarters of study participants reported high satisfaction with the OG physical exercise components of treatment, while participants reporting severe pain were more likely to drop out of the study. Experience with the iPad varied widely within the arms of this study. The main complaints were not related to the technology per se, but rather how the technology was performing due to connectivity issues such as poor Wi-Fi signal for some of the participants. This prevented a few participants from full participation in the televideo sessions or made operating the mHealth app more cumbersome (i.e., the activity tracker would not synchronize with the app or videos would run slower or with pauses). Indeed, participants did enjoy using the blood pressure monitor and were more tolerant when experiencing technical issues with this device because they clearly understood that the information it provided was important (note, the majority of these patients were taking antihypertensive medications). This underscores the importance of clearly explaining why certain devices and exercises are being used in the study to participants, since insufficient information combined with technical difficulties resulted in frustration.

Impressively, many of those who completed treatment reported that they continued to perform the exercises they had learned after they finished the study, with at least twice a week engagement in those activities. Exercise routines for older adults may require more than 12 weeks of training, particularly if the exercises are new $[35,36]$. Some evidence indicates that older adults feel more comfortable doing things they enjoy and have already experienced in the past [37], so interventions that modify existing exercise 
patterns are likely advisable for increasing adherence to physical regimens in this patient population. Another positive observation was the enthusiasm reported by the older adults who learned that other people in their community were participating in the study. Social support and social connection are highly important for all age groups but may be particularly so for older adults engaging in novel activities to sustain motivation [38]. Because most activities were conducted individually and within the home, we might have increased satisfaction and retention if we had included opportunities to engage in treatment components with other participants, perhaps virtually and in group settings.

Study Limitations / Identified Issues of Treatment Implementation. Limitations of this study include its small sample size, limited representation of participants from minority and rural populations, limited follow-up periods post intervention, and issues with technology in terms of internet connectivity. We encountered problems that were related to the community-based nature of our implementation, including issues of internet connectivity. This issue seemed to drive dropout, despite the fact that the study team tried to provide solutions for connectivity issues. Because the Otago component of the treatment is more commonly used in residential settings [31], it will be important to anticipate and prevent these problems in future studies with lower-income older adults to avoid negative experiences or discontinuation.

We also perceived that our participants may not have been able to form fully effective therapeutic relationships with our young therapists in Arm 3 to the extent that an older provider might have. Indeed, participants expressed this to our therapists and noted the large gap in age (of about 50 years). The preference of older adults with respect to matching with a provider similar to themselves appeared to extend beyond age, to also include preferences for matching in terms of appearance, gender and culture. Specifically, a recurring theme our team noted was participant lack of engagement with the GYYB component of treatment and with Behavioral Activation therapists. As this was a small, localized sample with limited representation of older adults, we believe that engagement may vary depending on the region of the country. Future considerations should include assurances that exercises are presented considering age, gender and cultural background similar to the specific target sample, to increase engagement with the activity.

The COVID-19 pandemic and subsequent public health advisory restrictions made it particularly difficult for older adults to engage with study personnel. The justifiable concern of older adults regarding close contact with study personnel such as during visits for resolving technical issues, or saliva sample collection was a major issue. Nonetheless, both the research team and participants showed extraordinary motivation to complete measures and to overcome these obstacles/restrictions, particularly concerning saliva sample collection (for example, use of additional protective gear). We believe this indicates that, under normal conditions, far fewer difficulties would be encountered. Unrelated to this, another limitation was underrepresentation of minority groups. Better representation would have been preferable.

Implications for Physical, Functional and Behavioral Health. Future studies of sufficient sample size should address lessons learned in this feasibility study, particularly with respect to attention to cultural / geographical issues, broadband connectivity issues, and efforts to increase social interaction during 
treatment components. Consideration of cultural and age representation, and appropriate language, for instance, using simpler wording to refer to activities for breathing or to support mood in video and print / tablet applications may increase engagement, participation, and adherence to treatment. Peers may also be a useful addition to treatment components and can help to address many of the aforementioned issues, such as resolving technical issues (to avoid frustration), personal contact to motivate oneself to complete the activities, or seeing/interacting with people they know in their community who successfully have completed treatment or know how to do the most challenging activities such as the GYYB or following the planned activities of Behavioral Activation. Finally, as pain was reportedly related to dropout in this small sample, future studies should consider implementing integrated strategies to address pain in combination with components of the Activate for Life treatment to increase retention. As COVID-19 was an unexpected event that challenged participants, research teams, and housing agencies, high levels of innovation and flexibility were brought to bear, and these modifications, along with mHealth and telehealth may help address the challenges noted in delivering this type of multidimensional treatment to older adults.

\section{Declarations}

\section{Ethics approval and consent to participate}

This study design was approved by the Medical University of South Carolina Institutional Review Board, approval \#Pro00076835 and registered at ClinicalTrials.gov Identifier NCT03853148. Signed informed consent was obtained from all participants contributing data to this study

\section{Consent for publication}

Not applicable

\section{Availability of data and materials}

Not applicable

\section{Competing interests}

The authors declare that they have no competing interests.

\section{Funding}

This award (P20 NR016575) was originally held by Dr. Kathryn VanRavenstein and is now overseen by Dr. Teresa Kelechi, the current Program Director of the MUSC SSMC; (2) the MUSC South Carolina Clinical \& Translational Research Institute (SCTR; UL1RR029882 and UL1TR000062). Funding for the research reported in this article was provided by (1) the National Institutes of Health / National Institute of Nursing Research (NIH / NINR) -supported Symptoms Self-Management Center (SSMC) located at the Medical University of South Carolina (MUSC). 


\section{Authors' contributions}

MAHT and TK conceptualized the study and authored most of the content of this manuscript. MAHT conducted the data analysis. AN, MM and SB completed critical review and edits of the manuscript. All the authors have read and approved the manuscript. The content of this manuscript is solely the responsibility of the authors and does not necessarily represent the official views of the NIH/NINR.

\section{Acknowledgements}

We would like to thank the older adults who have participated in this project and the support of the leadership and staff of the community living complex.

\section{Authors' information (optional)}

Melba A. Hernandez-Tejada, PhD, DHA

Department of Psychiatry and Behavioral Sciences, McGovern Medical School, The University of Texas Health Science Center, Houston, Texas, United States of America

Alexis Nagel, PhD

College of Nursing, Medical University of South Carolina, Charleston, South Carolina, United States of America

Mohan Madisetti BSc, MSc

College of Nursing, Medical University of South Carolina, Charleston, South Carolina, United States of America

Sundar Balasubramanian PhD

Department of Radiation Oncology, College of Medicine, Medical University of South

Carolina, Charleston, South Carolina, United States of America

Teresa Kelechi,'PhD, RN, FAAN

College of Nursing, Medical University of South Carolina, Charleston, South Carolina, United States of America

\section{References}

1. Mather M, Scommegna P, Kilduff L. Aging in the United States. [Internet]. 2019 Jul 19 [cited June1 2021]. Available from: https://www.prb.org/resources/fact-sheet-aging-in-the-united-states/ 
2. Stolz E, Mayerl H, Waxenegger A, Rásky É, Freidl W. Impact of socioeconomic position on frailty trajectories in 10 European countries: evidence from the Survey of Health, Ageing and Retirement in Europe (2004-2013). J Epidemiol Community Health. 2017 Jan 1;71(1):73-80. doi:10.1136/jech2016-207712.

3. Cubanski J, Koma W, Damico A, Neuman T, Kaiser Family Foundation. How Many Seniors Live in Poverty [Internet]. 2018 Nov 19. [cited 2021 Jun 1]. Available from: https://www.kff.org/medicare/issue-brief/how-many-seniors-live-in-poverty/

4. Li Z, Dalaker J. Poverty Among the Population Aged 65 and Older. Congressional Research Service Report. [Internet]. 2021 Apr 14 [cited Accessed 2021 Jun 1]. Available from https://crsreports.congress.gov/product/details?prodcode=R45791

5. Salminen M, Laine J, Vahlberg T, Viikari P, Wuorela M, Viitanen M, Viikari L. Factors associated with institutionalization among home-dwelling patients of Urgent Geriatric Outpatient Clinic: a 3-year follow-up study. European Geriatric Medicine. 2020 Oct;11:745-51. doi:10.1007/s41999-020-003387.

6. Ahn M, Kang J, Kwon HJ. The concept of aging in place as intention. The Gerontologist. 2020 Jan 24;60(1):50-9. doi:10.1093/geront/gny167.

7. Wiles JL, Leibing A, Guberman N, Reeve J, Allen RE. The meaning of "aging in place" to older people. The gerontologist. 2012 Jun 1;52(3):357 - 66. doi:10.1093/geront/gnr098.

8. Dahlhamer J, Lucas J, Zelaya C, Nahin R, Mackey S, DeBar L, Kerns R, Von Korff M, Porter L, Helmick C. Prevalence of chronic pain and high-impact chronic pain among adults-United States, 2016. Morbidity and Mortality Weekly Report. 2018 Sep 14;67(36):1001. doi: http://dx.doi.org/10.15585/mmwr.mm6736a2.

9. Meng H, Hale L, Friedberg F. Prevalence and predictors of fatigue among middle-aged and older adults: evidence from the Health and Retirement study. Journal of the American Geriatrics Society. 2010 Oct;58(10):2033. doi:10.1111/j.1532-5415.2010.03088.x.

10. Butera KA, Roff SR, Buford TW, Cruz-Almeida Y. The impact of multisite pain on functional outcomes in older adults: biopsychosocial considerations. Journal of pain research. 2019;12:1115. doi:10.2147/JPR.S192755.

11. Cai $Y$, Leveille SG, Shi L, Chen $P$, You T. Chronic pain and risk of injurious falls in community-dwelling older adults. The Journals of Gerontology: Series A. 2021 Sep;76(9):e179-86. doi:10.1093/gerona/glaa249.

12. Domenichiello AF, Ramsden CE. The silent epidemic of chronic pain in older adults. Progress in Neuro-Psychopharmacology and Biological Psychiatry. 2019 Jul 13;93:284-90. doi:10.1016/j.pnpbp.2019.04.006.

13. Rai R, Jongenelis MI, Jackson B, Newton RU, Pettigrew S. Factors influencing physical activity participation among older people with low activity levels. Ageing Society. 2020 Dec;40(12):2593613. doi:https://doi.org/10.1017/S0144686X1900076X. 
14. Ho LY, Ng SS. Non-pharmacological interventions for fatigue in older adults: a systematic review and meta-analysis. Age and ageing. 2020 Apr 27;49(3):341 - 51. doi:10.1093/ageing/afaa019.

15. Kechichian A, Lafrance S, Matifat E, Dubé F, Lussier D, Benhaim P, Perreault K, Filiatrault J, Rainville P, Higgins J, Rousseau J. Multimodal Interventions Including Rehabilitation Exercise for Older Adults With Chronic Musculoskeletal Pain: A Systematic Review and Meta-analyses of Randomized Controlled Trials. Journal of Geriatric Physical Therapy. 2020 Aug 6. doi: 10.1519/JPT.0000000000000279.

16. Bantham A, Ross SE, Sebastião E, Hall G. Overcoming barriers to physical activity in underserved populations. Prog Cardiovasc Dis. 2020 Nov 5. doi:10.1016/j.pcad.2020.11.002.

17. Osborn R, Doty MM, Moulds D, Sarnak DO, Shah A. Older Americans were sicker and faced more financial barriers to health care than counterparts in other countries. Health Affairs. $2017 \mathrm{Dec}$ 1;36(12):2123-32. doi:10.1377/hlthaff.2017.1048.

18. Changizi $\mathrm{M}$, Kaveh MH. Effectiveness of the $\mathrm{mHealth}$ technology in improvement of healthy behaviors in an elderly population-A systematic review. Mhealth. 2017;3. doi:10.21037/mhealth.2017.08.06.

19. Pho AT, Tanner EK, Roth J, Greeley ME, Dorsey CD, Szanton SL. Nursing strategies for promoting and maintaining function among community-living older adults: the CAPABLE intervention. Geriatric nursing. 2012 Nov 1;33(6):439 - 45. doi:10.1016/j.gerinurse.2012.04.002.

20. Acierno R, Knapp R, Tuerk P, Gilmore AK, Lejuez C, Ruggiero K, Muzzy W, Egede L, Hernandez-Tejada MA, Foa EB. A non-inferiority trial of prolonged exposure for posttraumatic stress disorder: in person versus home-based telehealth. Behav Res Ther. 2017 Feb;1:89:57-65.

21. Greenwald P, Stern ME, Clark S, Sharma R. Older adults and technology: in telehealth, they may not be who you think they are. International journal of emergency medicine. 2018 Dec;11(1):1-4. doi:10.1186/s12245-017-0162-7.

22. Kruse C, Fohn J, Wilson N, Patlan EN, Zipp S, Mileski M. Utilization barriers and medical outcomes commensurate with the use of telehealth among older adults: systematic review. JMIR medical informatics. 2020;8(8):e20359. doi:10.2196/20359.

23. Balasubramanian S. Gentle Yoga \& Yogic Breathing for Scleroderma Symptom Management [video on the internet] 2020. Available from: https://www.youtube.com/watch?v=nhAGBMCvtlY.

24. Lejuez CW, Hopko DR, Acierno R, Daughters SB, Pagoto SL. Ten year revision of the brief behavioral activation treatment for depression: revised treatment manual. Behav Modif. 2011 Mar;35(2):11161. doi:10.1177/0145445510390929.

25. Stevens JA, Burns E. A CDC compendium of effective fall interventions: What works for communitydwelling older adults. 2015 [cited 2021 June 1]. Available from: https://stacks.cdc.gov/view/cdc/30689

26. Borson S, Scanlan JM, Chen P, Ganguli M. The Mini-Cog as a screen for dementia: validation in a population-based sample. J Am Geriatr Soc. 2003 Oct;51(10):1451-4.

27. doi:10.1046/j.1532-5415.2003.51465.x. 
28. Linardon J, Fuller-Tyszkiewicz M. Attrition and adherence in smartphone-delivered interventions for mental health problems: A systematic and meta-analytic review. J Consult Clin Psychol. 2020 Jan;88(1):1. doi:10.1037/ccp0000459.

29. Torous J, Lipschitz J, Ng M, Firth J. Dropout rates in clinical trials of smartphone apps for depressive symptoms: a systematic review and meta-analysis. Journal of affective disorders. $2020 \mathrm{Feb}$ 15;263:413-9. doi:10.1016/j.jad.2019.11.167.

30. Cramer H, Haller H, Dobos G, Lauche R. A systematic review and meta-analysis estimating the expected dropout rates in randomized controlled trials on yoga interventions. Evidence-Based Complementary and Alternative Medicine. 2016 Jan 1;2016. doi:10.1155/2016/5859729.

31. Inzitari M, Pérez LM, Enfedaque MB, Soto L, Díaz F, Gual N, Martín E, Orfila F, Mulero P, Ruiz R, Cesari $M$. Integrated primary and geriatric care for frail older adults in the community: implementation of a complex intervention into real life. European journal of internal medicine. 2018 Oct 1;56:57-63. doi:10.1016/j.ejim.2018.07.022.

32. Weber M, Belala N, Clemson L, Boulton E, Hawley-Hague H, Becker C, Schwenk M. Feasibility and effectiveness of intervention programmes integrating functional exercise into daily life of older adults: a systematic review. Gerontology. 2018;64(2):172-87. doi:10.1159/000479965.

33. Watanabe Y, Yamada Y, Yoshida T, Yokoyama K, Miyake M, Yamagata E, Yamada M, Yoshinaka Y, Kimura M. Kyoto-Kameoka Study Group. Comprehensive geriatric intervention in communitydwelling older adults: a cluster-randomized controlled trial. Journal of cachexia, sarcopenia and muscle. 2020 Feb;11(1):26-37. doi:10.1002/jcsm.12504.

34. Wingert JR, Corle CE, Saccone DF, Lee J, Rote AE. Effects of a Community-Based Tai Chi Program on Balance, Functional Outcomes, and Sensorimotor Function in Older Adults. Physical \& Occupational Therapy In Geriatrics. 2020 Apr 2;38(2):129 - 50. doi: 10.1080/02703181.2019.1709600.

35. Vailati Riboni F, Comazzi B, Bercovitz K, Castelnuovo G, Molinari E, Pagnini F. Technologicallyenhanced psychological interventions for older adults: a scoping review. BMC Geriatr. 2020 Dec;20:1-1. doi:10.1186/s12877-020-01594-9.

36. Langhammer B, Bergland A, Rydwik E. The importance of physical activity exercise among older people.2018:7856823. doi:10.1155/2018/7856823.

37. Wallace R, Lees C, Minou M, Singleton D, Stratton G. Effects of a 12-week community exercise programme on older people. Nursing older people. 2014 Jan 31;26(1). doi:10.7748/nop2014.02.26.1.20.e508.

38. Graham LJ, Connelly DM. "Any movement at all is exercise": A focused ethnography of rural community-dwelling older adults' perceptions and experiences of exercise as self-care. Physiotherapy Canada. 2013 Oct;65(4):333-41. doi:10.3138/ptc.2012-31.

39. Palmer SJ. Encouraging exercise in older adults: advice for nurses. British journal of community nursing. 2020 Feb 2;25(2):95 - 7. doi:10.12968/bjcn.2020.25.2.95.

\section{Figures}




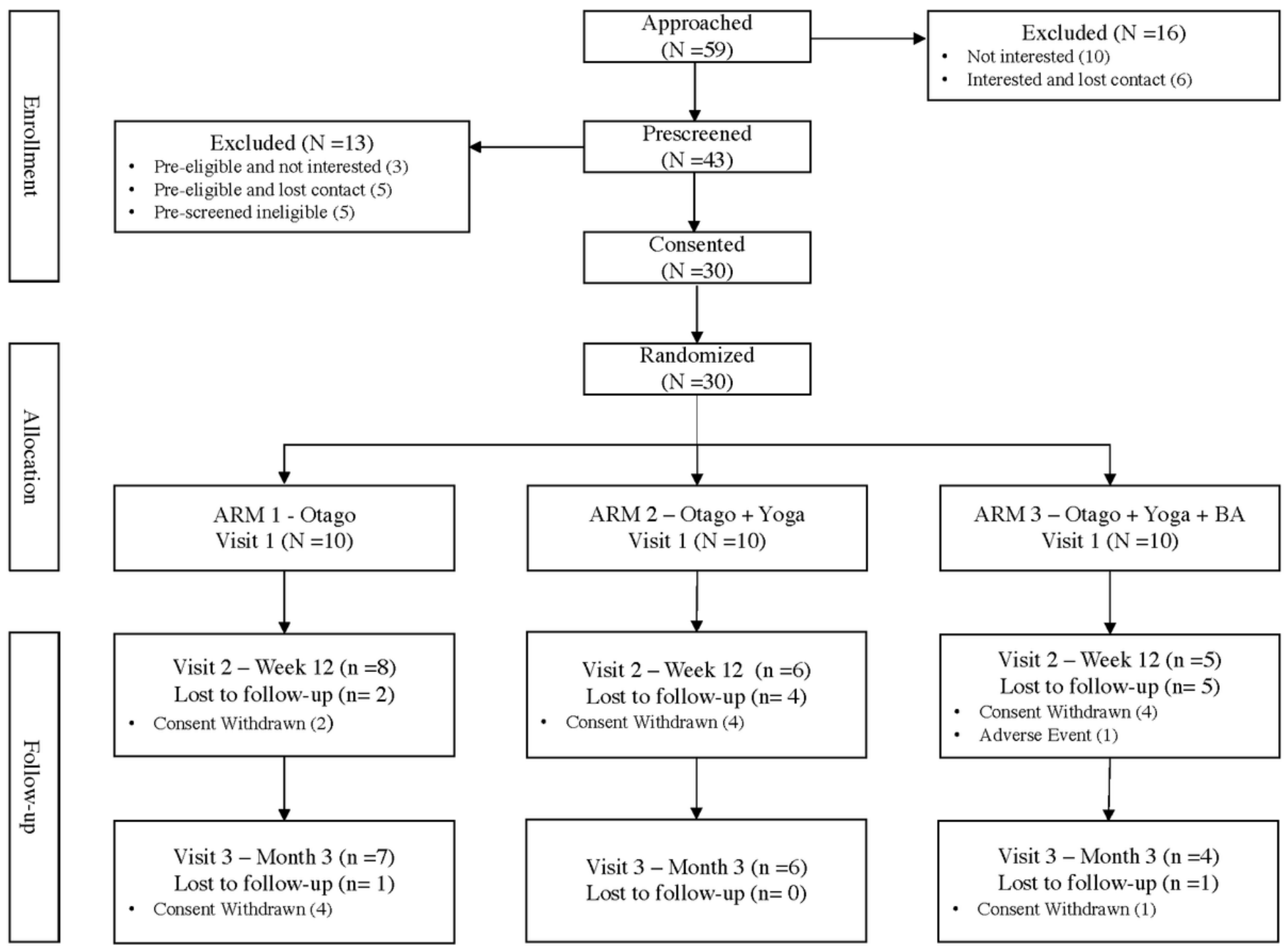

\section{Figure 1}

Consort Diagram 